\title{
Incidence of urticaria, angioedema, and type I hypersensitivity reactions associated with fibrinolytic agents in Thailand using the database of the health product vigilance center
}

\author{
Hutsaya Prasitdumrong, ${ }_{1}^{1}$ Kawin Duangmee, ${ }^{2}$ Pornwalai Boonmuang, ${ }^{3}$ Wichai Santimaleeworagun, ${ }^{3}$ Yaowares Oppamayun, \\ Cholticha Sonsupap, ${ }^{4}$ Thawatchai Nakkaratniyom ${ }^{4}$
}

\begin{abstract}
Background: Besides hemorrhage, allergic reactions have also been observed in several clinical trials of fibrinolytic agents. These reactions might negatively affect patient outcomes, especially life-threatening type I hypersensitivity reactions such as anaphylaxis. However, there are limited data on the incidence of these reactions.
\end{abstract}

Objective: The aim of study was to analyze the incidence of urticaria, angioedema, and type I hypersensitivity reactions from fibrinolytic agents for various indications.

Methods: A retrospective analysis of data from the Thai Vigibase database was conducted. All reports of adverse drug reactions from fibrinolytic agents from 1984 to 2017 were identified using the World Health Organization adverse reaction terminology. The proportion of each suspected adverse drug reaction and the cumulative incidence were calculated.

Results: A total of 284 reports were identified in the Thai Vigibase database. The overall incidence of urticaria, angioedema, and type I hypersensitivity reactions for the streptokinase group was 52.64/10,000 persons, with individual incidence rates of $9.64 / 10,000$ persons for urticaria, $8.90 / 10,000$ persons for angioedema, and 34.11/10,000 persons for type I hypersensitivity reactions. In the alteplase group, the overall incidence for all suspected reactions was $18.90 / 10,000$ persons, with individual incidence rates of 3.29/10,000 persons for urticaria, 5.75/10,000 persons for angioedema, and 9.86/10,000 persons for type I hypersensitivity reactions.

Conclusion: Type I hypersensitivity reactions were the most common allergic reactions from fibrinolytic agents. It is necessary to take these reactions into consideration when using fibrinolytic therapy.

Key words: anaphylaxis, hypotension, streptokinase, tenecteplase, tissue plasminogen activator

\section{Citation:}

Prasitdumrong, H., Duangmee, K., Boonmuang, P., Santimaleeworagun, W., Oppamayun, Y., Sonsupap, C.,

Nakkaratniyom, T. (2023) Incidence of urticaria, angioedema, and type I hypersensitivity reactions associated

with fibrinolytic agents in Thailand using the database of the health product vigilance center. Asian Pac J Allergy Immunol, 41(1), 67-72. https://doi.org/10.12932/ap-181119-0694

\section{Affiliations:}

${ }^{1}$ Cardiovascular Unit, Department of Internal Medicine,

Phramongkutklao Hospital, Bangkok, Thailand

2 The College of Pharmacotherapy of Thailand, The Pharmacy Council, Nonthaburi, Thailand

${ }^{3}$ Department of Pharmacy, Faculty of Pharmacy, Silpakorn University, Nakhon Pathom, Thailand

${ }^{4}$ Health Product Vigilance Center, Technical and Planning Division, Food and Drug Administration, Ministry of Public Health, Thailand

Corresponding author:

Pornwalai Boonmuang

Faculty of Pharmacy, Silpakorn University

Sanam Chandra Palace Campus. No. 6 RajamankhaNai Road, PhraPathom Chedi, Muang, Nakorn Pathom 73000, Thailand

E-mail: boonmuang_p@su.ac.th 


\section{Introduction}

Fibrinolytic therapy is an important treatment strategy for various indications including acute myocardial infarction (AMI), acute ischemic stroke, massive pulmonary embolism (PE) and arterial or vein thrombosis. ${ }^{1}$ However, several controlled trials have reported hypersensitivity reactions with the use of fibrinolytic agents, in addition to bleeding complications. Streptokinase, which possesses antigenicity properties, was found to cause hypersensitivity reactions in $5 \%$ of cases, including anaphylaxis in $0.6 \%{ }^{2,3}$ Surprisingly, hypersensitivity reactions were also reported for fibrin-specific fibrinolytic agents in some clinical trials. For example, recombinant tissue plasminogen activator (rt-PA), also known as alteplase, was found to cause hypersensitivity reactions in $1.5 \%$ of cases, including anaphylaxis in $0.2 \%$, similar to that of tenecteplase (TNK). ${ }^{4-6}$ These events are classified as type I hypersensitivity reactions, which induce an immediate response, indicate worsening clinical outcomes, and can be life threatening. The mechanism underlying these reactions is still unclear; however, activation of complement cascade system and the kinin pathway resulting in bradykinin congestion have been proposed. ${ }^{7}$

Controlled clinical trials may not manifest the real condition of patients, who may have more clinical complications owing to strict inclusion or exclusion criteria and fewer participants. Conducting a pharmacovigilance study is one of the most important strategies to contribute real-world evidence. The Health Product Vigilance Center (HPVC) was established in 1983 under the Thai Food and Drug Administration, Ministry of Public Health (http:// thaihpvc.fda.moph.go.th). The responsibilities of HPVC include monitoring and reporting adverse drug reactions (ADRs) from all health products, including medications, in Thailand. The spontaneous reporting system is one of the HPVC systems that collects nationwide adverse reaction reports from health care professionals. More than 45,000 reports recorded in 2017 are included in the Thai Vigibase database, which contains over 660,000 reports in total, of which 886 are associated with the use of fibrinolytic agents. ${ }^{8}$ Therefore, the aims of this retrospective, descriptive, naturalistic study were to analyze the incidence of urticaria, angioedema, and type I hypersensitivity reactions and report the characteristics of patients who suffered these reactions using the Thai Vigibase database.

\section{Methods}

The Thai Vigibase database includes all reports of suspected adverse drug events submitted by health care providers from January 1, 1984, through December 31, 2017. Institutional Review Board approval (Q021h/61_Exp) was obtained to retrospectively review data from the Thai Vigibase. The clinical trial registration number was TCTR20191030004, which was approved by the Thai Clinical Trials Registry.
All reports of urticaria, angioedema, or type I hypersensitivity reactions due to streptokinase, alteplase, or TNK were included. Terms specified in the spontaneous reporting system according to the WHO adverse reaction terminology (WHO-ART) were used for identifying the reactions. "Urticaria" and "wheals" were used to identify urticaria. For angioedema, the terms were "angioedema," "face oedema," "lips swelling nonspecific," "oedema eyelid," "oedema mouth," "oedema periorbital," and "tongue swelling non-specific." We classified type I hypersensitivity reactions into the following three categories: anaphylactic reaction, hemodynamic complication, and respiratory complication. An anaphylactic reaction was identified by the terms "anaphylactic reaction," "anaphylactic shock," "anaphylactoid reaction," and "anaphylaxis." Hemodynamic complications were identified by the terms "arterial blood pressure decreased," "blood pressure drop arterial," "hypotension," "shock cardiogenic", and "syncope." Finally, the terms "breathing arrested," "breathing difficult," "chest discomfort," "fullness of chest," "tightness of chest," "dyspnoea," and "wheezes" were used to identify respiratory complications. Any reports described as "unlikely" based on the Naranjo probability scale (Appendix A) ${ }^{9}$ were excluded.

From each report, the following details were extracted: gender, age, history of medication allergy, comorbidities, laboratory data, concomitant medications, dosage and administration, indication, first and last date of fibrinolytic agent use, suspected ADR and onset, severity, Naranjo probability scale score, clinical outcomes, and outcomes from dechallenge or rechallenge.

The proportion of each suspected ADR was calculated as the number of reports for each suspected ADR divided by the total number of suspected ADRs reported for each fibrinolytic agent, expressed as a percentage.

The cumulative incidences were calculated separately for new events of urticaria, angioedema, type I hypersensitivity reactions, anaphylactic reactions, hypotension, and respiratory complications reported from January 1, 2013, through December 31, 2017. Moreover, the total number of urticaria, angioedema, and type I hypersensitivity reactions were also calculated. Any duplicated patients with different adverse drug reaction reports were excluded from the incidence calculation, whereby only the first case when a reaction occurred was selected. The denominator used to calculate the incidence was the number of fibrinolytic-exposed patients. This information was obtained from the national health security schemes database, which covers $95.5 \%$ of the population, representing almost the entire Thai population except for self-insured individuals. ${ }^{10}$ ICD-10 codes were used for the identification and categorization of each indication as follows: AMI was defined as I21 (AMI), I24 (other acute ischemic heart diseases), or I46 (cardiac arrest); acute ischemic stroke was defined as I63 (cerebral infarction), I64 (stroke, not specified) or I65 (occlusion and stenosis of precerebral arteries, not resulting in cerebral infarction); pulmonary embolism was defined as I26; and an artery or vein thrombosis was defined as I74. 
Data management was performed using Microsoft Excel (Microsoft Corporation, Redmond, WA, USA). Descriptive analyses were used to examine differences among the fibrinolytic groups for mean age, gender proportion, Naranjo probability scale score, severity, clinical outcomes, history of medication allergy, suspected ADRs, and outcomes from dechallenge or rechallenge. Fisher's exact test and chi-square test were used for all statistical analyses, conducted using STATA $^{\circledast}$ version 15.1 (Stata Corp, College Station, TX, USA).

\section{Results}

The first case of a type I hypersensitivity reaction to streptokinase was reported in 2000. A total of 284 reports of urticaria, angioedema, and type I hypersensitivity reactions were identified, of which 242 and 42 reports were for patients exposed to streptokinase and alteplase, respectively. All four reports of reactions to tenecteplase were excluded.

Table 1. Demographic and clinical characteristics of 264 fibrinolytic-associated urticaria, angioedema, and type I hypersensitivity reactions

\begin{tabular}{|c|c|c|c|}
\hline \multirow[b]{2}{*}{ Parameter } & \multicolumn{3}{|c|}{ Number of patients (\%) $[\mathrm{N}=264]$} \\
\hline & $\begin{array}{l}\text { Streptokinase } \\
\qquad[\mathbf{N}=230]\end{array}$ & $\begin{array}{c}\text { Alteplase } \\
{[\mathbf{N}=34]}\end{array}$ & $P$-value \\
\hline \multicolumn{4}{|l|}{ Age (years) } \\
\hline Mean \pm SD & $61.31 \pm 12.67$ & $62.48 \pm 12.88$ & \\
\hline $15-30$ & $1(0.43)$ & $1(2.94)$ & \\
\hline $31-45$ & $17(7.39)$ & $1(2.94)$ & \\
\hline $46-60$ & $70(30.43)$ & $12(35.29)$ & 0.608 \\
\hline $61-75$ & $73(31.74)$ & $13(38.23)$ & \\
\hline $76-90$ & $29(12.61)$ & $4(11.76)$ & \\
\hline 90 up & $1(0.43)$ & 0 & \\
\hline Not reported & $39(16.97)$ & $3(8.82)$ & \\
\hline \multicolumn{4}{|l|}{ Gender } \\
\hline Male & $168(73.05)$ & $18(52.94)$ & $0.026^{*}$ \\
\hline Female & $61(26.52)$ & $15(44.12)$ & \\
\hline Not reported & $1(0.43)$ & $1(2.94)$ & \\
\hline \multicolumn{4}{|l|}{$\begin{array}{l}\text { History of medication } \\
\text { allergy }\end{array}$} \\
\hline No known allergy & $224(97.39)$ & $33(97.06)$ & \\
\hline Known allergy & $6(2.61)$ & $1(2.94)$ & \\
\hline \multicolumn{4}{|l|}{ Comorbidities } \\
\hline Not reported & $219(95.22)$ & $22(64.71)$ & \\
\hline Known comorbidities & $11(4.78)$ & $12(35.29)$ & \\
\hline \multicolumn{4}{|l|}{ Concomitant medications } \\
\hline Not reported & $202(87.83)$ & $31(91.18)$ & \\
\hline $\begin{array}{l}\text { Received other } \\
\text { medications }\end{array}$ & $28(12.17)$ & $3(8.82)$ & \\
\hline
\end{tabular}

SD standard deviation

${ }^{*}$ Chi square test, level of significant $p<0.05$
Table 1 provides the demographic and clinical characteristics of the reported cases. The five most common comorbidities identified among the patients were hypertension, diabetes mellitus, ischemic heart disease or history of myocardial infarction, history of any cerebrovascular accident, and atrial fibrillation. However, of the concomitant medications and comorbidities, hypertension was found in $23.53 \%$ of the patients who received alteplase. The most common indication for streptokinase use was AMI, whereas alteplase was widely used for acute ischemic stroke at a mean total dose of $55.41 \mathrm{mg}$.

There were 26, 31, and 227 reports related to urticaria, angioedema, and type I hypersensitivity reactions, respectively. The proportion of anaphylactic reactions among the suspected ADRs was $47.62 \%$ of patients in the alteplase group and $9.09 \%$ of patients in the streptokinase group $(p<0.001)$, and anaphylactoid reactions were reported in $2.38 \%$ and $0.83 \%$ of patients, respectively, $(p=0.34)$. The number of reports of angioedema was significantly higher in the alteplase group $(21.44 \%)$ than in the streptokinase group (9.09\%; $p=0.012)$; however, in both groups, most reports did not specify the location. Tongue (two reports, $4.76 \%$ ) and lip and periorbital (one report each, 2.38\%) locations were reported in the alteplase group. In contrast, patients in the streptokinase group had a higher rate of hypotension (70.66\% compared to $11.9 \%$ in the alteplase group; $p<0.001$ ), and only $0.83 \%$ reported syncope. The rates of urticaria and respiratory complications (e.g., dyspnea, chest discomfort) did not differ between the two groups (Table 2).

Table 2. Number and proportion ${ }^{\dagger}$ of suspected adverse drug reactions reports from streptokinase and alteplase

\begin{tabular}{|l|c|c|c|}
\hline \multirow{2}{*}{\multicolumn{1}{|c|}{ Parameter }} & \multicolumn{3}{|c|}{ Number (Proportion, \%) [N = 284] } \\
\cline { 2 - 4 } & $\begin{array}{c}\text { Streptokinase } \\
{[\mathbf{N}=\mathbf{2 4 2}]}\end{array}$ & $\begin{array}{c}\text { Alteplase } \\
{[\mathbf{N}=42]}\end{array}$ & P-value \\
\hline Urticaria & $22(9.09)$ & $4(9.52)$ & 0.756 \\
\hline Angioedema & $22(9.09)$ & $9(21.44)$ & $0.012^{*}$ \\
\hline $\begin{array}{l}\text { Type I hypersensitivity } \\
\text { reactions }\end{array}$ & $22(9.09)$ & $20(47.62)$ & $<0.001^{*}$ \\
\hline $\begin{array}{l}\text { Anaphylactic reactions } \\
\text { Hypotension }\end{array}$ & $171(70.66)$ & $5(11.9)$ & $<0.001^{*}$ \\
\hline $\begin{array}{l}\text { Respiratory } \\
\text { complications }\end{array}$ & $5(2.07)$ & $4(9.52)$ & 0.130 \\
\hline
\end{tabular}

${ }^{\dagger}$ Calculated with the number of each suspected ADR reports divided by the number of total suspected ADR reports.

${ }^{*}$ Defined as Breathing arrested, Chest discomfort, Chest fullness, Chest tightness, Dyspnea, Wheezes

${ }^{*}$ Chi square test, level of significant $p<0.05$ 


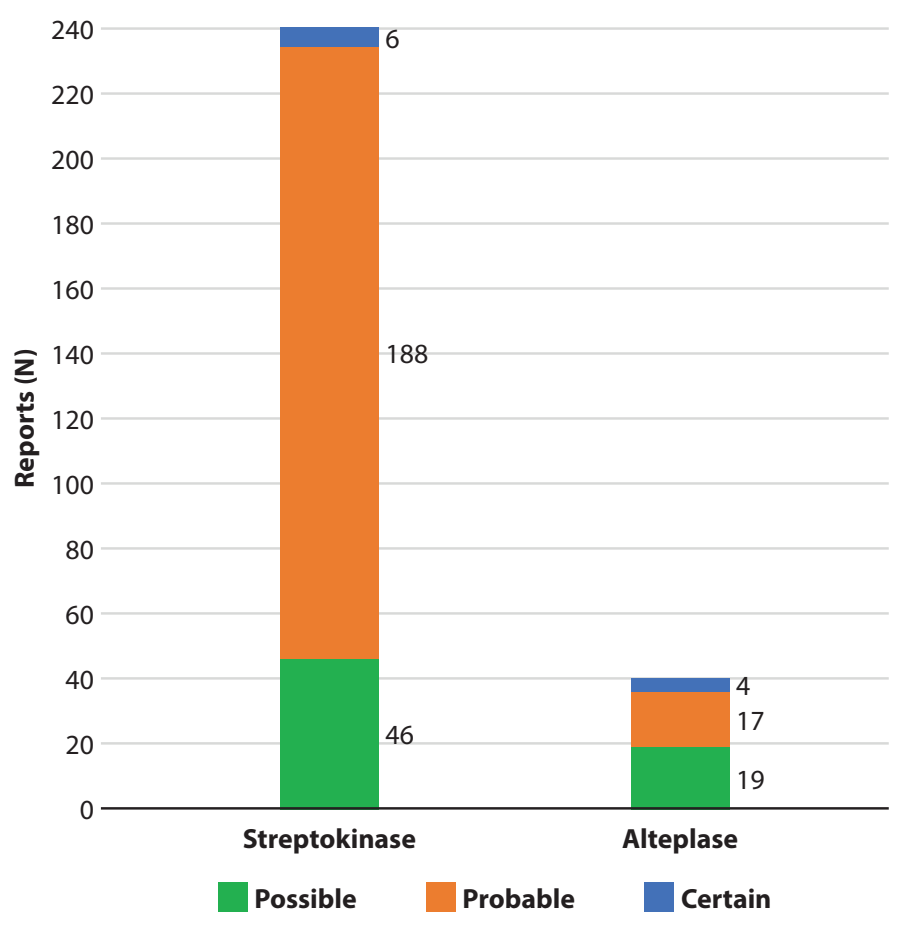

Figure 1. Naranjo probability scale of all suspected adverse drug reactions
The time-to-onset of these ADRs was usually within $24 \mathrm{~h}$; however, some reports showed a delay in onset to between 24 and $48 \mathrm{~h}$. Based on the Naranjo probability scale, the causality of the majority of reports was categorized as "probable" (Figure 1).

Major outcomes included complete recovery, although several reports were classified as severe. In the streptokinase group, death was recorded as the clinical outcome in four reports of type I hypersensitivity reactions (1.65\%). In addition, we found that $21.74 \%$ of patients in the streptokinase group and $11.76 \%$ in the alteplase group were rechallenged but only $2 \%$ of them had experienced ADRs. Rechallenge was necessary in these patients because they required treatment with fibrinolytic agents.

Table 3 summarizes the cumulative incidence of suspected ADRs in this study. In the streptokinase group, hypotension showed the highest incidence for overall indications and for AMI. In the alteplase group, anaphylactic reactions showed the highest incidence for overall indications and for acute ischemic stroke. However, the incidence rates of $\mathrm{PE}$ and arterial or vein thrombosis were not calculated due to the small number of reports.

Table 3. The cumulative incidence of urticaria, angioedema, and type I hypersensitivity reactions in streptokinase and alteplase groups during 2013 to 2017.

\begin{tabular}{|c|c|c|c|c|c|c|c|c|}
\hline \multirow{3}{*}{ Adverse drug reactions } & \multicolumn{4}{|c|}{ Streptokinase group } & \multicolumn{4}{|c|}{ Alteplase group } \\
\hline & \multicolumn{2}{|c|}{$\begin{array}{c}\text { Overall } \\
{[n=13,487]}\end{array}$} & \multicolumn{2}{|c|}{$\begin{array}{c}\text { AMI } \\
{[n=13,362]}\end{array}$} & \multicolumn{2}{|c|}{$\begin{array}{c}\text { Overall } \\
{[n=12,172]}\end{array}$} & \multicolumn{2}{|c|}{$\begin{array}{l}\text { Acute ischemic stroke } \\
\qquad[\mathrm{n}=11,638]\end{array}$} \\
\hline & $\begin{array}{l}\text { Cases } \\
\text { (n) }\end{array}$ & $\begin{array}{l}\text { CIs (per } 10,000 \\
\text { patients) }\end{array}$ & $\begin{array}{l}\text { Cases } \\
(\mathbf{n})\end{array}$ & $\begin{array}{l}\text { CIs (per } 10,000 \\
\text { patients) }\end{array}$ & $\begin{array}{l}\text { Cases } \\
(\mathbf{n})\end{array}$ & $\begin{array}{l}\text { CIs (per } 10,000 \\
\text { patients) }\end{array}$ & $\begin{array}{l}\text { Cases } \\
\text { (n) }\end{array}$ & $\begin{array}{c}\text { CIs (per } 10,000 \\
\text { patients) }\end{array}$ \\
\hline $\begin{array}{l}\text { Composite of urticaria, angioedema, } \\
\text { and type I hypersensitivity reactions }\end{array}$ & 71 & 52.64 & 57 & 42.66 & 23 & 18.90 & 17 & 14.16 \\
\hline Urticaria & 13 & 9.64 & 8 & 5.99 & 4 & 3.29 & 3 & 2.58 \\
\hline Angioedema & 12 & 8.90 & 8 & 5.99 & 7 & 5.75 & 6 & 5.16 \\
\hline Type I hypersensitivity reactions & 46 & 34.11 & 41 & 30.68 & 12 & 9.86 & 8 & 6.87 \\
\hline Anaphylactic reactions & 5 & 3.71 & 2 & 1.50 & 8 & 6.57 & 5 & 4.30 \\
\hline Hypotension & 39 & 28.92 & 38 & 28.44 & 2 & 1.64 & 2 & 1.72 \\
\hline Respiratory complications $s^{\ddagger}$ & 2 & 1.48 & 1 & 0.75 & 2 & 1.64 & 1 & 0.86 \\
\hline
\end{tabular}

CIs cumulative incidences, AMI acute myocardial infarction

${ }^{*}$ Defined as Breathing arrested, Chest discomfort, Chest fullness, Chest tightness, Dyspnea, Wheezes 


\section{Discussion}

This pharmacovigilance study provides significant data compared to controlled clinical trials as it is representative of patients in real-world conditions. However, studies on fibrinolytic agents are currently scarce, and none have focused on allergic reactions. To our knowledge, this is the first study to analyze data pertaining to urticaria, angioedema, and type I hypersensitivity reactions caused by fibrinolytic agents for various indications.

Betancourt et al. ${ }^{11}$ reported pharmacovigilance data from 1660 patients with AMI who received streptokinase in Cuba. The authors observed that among a total of 792 case reports of ADRs, there were 285 cases of hypotension (36\%), which was the most frequent $\mathrm{ADR}$, and 47 cases of allergy (5.9\%), which primarily comprised skin rashes (72\%); however, they did not report any type I hypersensitivity reactions. Our pharmacovigilance study was quite different in design compared to that of Betancourt and colleagues. For instance, their study used a prospective method focusing on AMI, unlike our study which included various indications. However, both were based on spontaneous reporting. The criteria for determining ADRs were also different.

The International Collaborative Study of Severe Anaphylaxis reported international data on the risk of anaphylaxis due to medication use in hospitals across eight countries. ${ }^{12}$ The authors of this study found that the incidence of anaphylaxis due to streptokinase use was 284/100,000 exposed patients (95\% CI $[61,1312])$. Patients with AMI or PE were excluded in this study, and the likelihood was mostly classified as "unlikely". However, the present study reported a lower incidence of anaphylaxis. The differences between studies might be explained by the inclusion of all indications of streptokinase in the current study, resulting in a larger denominator compared to the previous study.

In general, alteplase is associated with fewer ADRs than those caused by streptokinase, including allergic reactions or hypotension due to the specific pharmacological action of fibrin. Data from several controlled trials on AMI have confirmed this hypothesis and reported rates of anaphylaxis, unspecified allergic reaction, and hypotension of $0.06 \%, 2 \%$, and $10-20 \%$, respectively, ${ }^{3,13-15}$ In contrast, the majority of trials on acute ischemic stroke rarely disclose these safety outcomes. The CASES prospective observational cohort study conducted across 60 centers in Canada assessed all participants who received intravenous alteplase for acute ischemic stroke $(\mathrm{n}=1135),{ }^{16}$ and found that 75 patients (6.6\%) experienced serious ADRs, with $1.3 \%$ of cases developing orolingual angioedema and $0.4 \%$ developing hypotension. No anaphylactic reactions were reported. However, in our study, hypotension occurred in $11.9 \%$ of cases and angioedema developed in $21.44 \%$, with orolingual angioedema accounting for $7.14 \%$ of cases. We also found one case of periorbital angioedema (2.38\%), which was not reported in the Canadian study. An interesting result was the astonishingly higher proportion and incidence of anaphylactic reactions. We hypothesize that there is a relationship between anaphylaxis and acute ischemic stroke, particularly in the brain stem region, notwithstanding limited data. ${ }^{17}$ The signs or symptoms of these reaction were difficult to differentiate, another diagnosis may have been proposed such as urticarial, which is often self-limited.

The characteristics of patients in this study are similar to those in previous research, including clinical trials and pharmacovigilance data from Western countries, in terms of the advanced age and female gender., ${ }^{3,11,13,14,18}$ In our study, we did not demonstrate a statistically significant association between the suspected ADRs and any age range; however, we identified a statistically significant association with gender. Nevertheless, males are frequently affected by diseases indicated for fibrinolytic agents, which may influenced this coincidental result.

In this study, the higher incidence of suspected ADRs than that observed in previous studies can be attributed to differences in calculated denominators and the criteria for ADRs, and that most reporters were pharmacists. All participants had Asian ancestry. Ethnic differences in terms of anaphylaxis incidence indicate that genetic modifiers can exist in human populations. For example, a retrospective study reported a higher incidence of anaphylaxis in South Asian than in white populations. ${ }^{19}$ Another pharmacovigilance study reported that anaphylaxis accounts for at least $12 \%$ of adverse drug events reported in China, ${ }^{20}$ which was higher than that reported in Cuba. ${ }^{11}$ Nevertheless, the reasons for these ethnic disparities remain unclear, but might reflect genetic diversity, socioeconomic status, or a multifactorial cause..$^{21}$

The Thai Vigibase database contains rare reports of patients who developed any allergic reaction from the use of fibrinolytic agents for massive PE, similar to that reported in controlled clinical studies. ${ }^{22,23}$ The reasons may be attributed to the use of a lower recommended dose than those used for AMI, accompanied by a slower infusion rate for administration.

The present retrospective study has some limitations. First, the spontaneous reporting system is a voluntary system, which results in under-reporting and poor documentation, especially concomitant medications or comorbidities, which may have affected our data analysis. Second, misdiagnosis may have occurred as the symptoms can be difficult to recognize and can be easily confused with cardiogenic shock, sudden death, or pulmonary edema. We could not differentiate these cases due to a lack of data. Third, we did not include data for patients with PE and artery or vein thrombosis. We also could not report the number of self-insured patients.

\section{Conclusion}

In conclusion, type I hypersensitivity reactions showed the highest incidence among the suspected ADRs based on anaphylactic reactions, and hypotension was a major variable in the streptokinase group. A remarkable result of this study was that alteplase treatment resulted in a higher rate of anaphylactic reactions and angioedema than streptokinase, although the incidence was low. Furthermore, this study showed no significant differences between urticaria and respiratory complications. The risk of these reactions must be carefully considered when deciding whether to use fibrinolytic therapy. 


\section{Acknowledgments}

The authors declare that there are no conflicts of interest associated with this work. No sources of funding were used to conduct this study or prepare this manuscript. We gratefully acknowledge the HPVC, Food and Drug Administration, Ministry of Public Health, Thailand, for providing support to data access and for their valuable inputs in the manuscript. The authors also want to thank the National Health Security Office (NHSO), Central Office for Healthcare Information (CHI), The Comptroller General's Department (CGD), and Social Security Office (SSO) for permitting to explore the national database. Furthermore, we particularly thank Miss Narisa Ruenroengboon for the beneficial recommendations in the statistical analysis of our study and Miss Jutatip Thungthong for the kind assistance in data collection. The authors would like to thank Enago (www. enago.com) for the English language review.

\section{References}

1. Srimahachota S, Boonyaratavej S, Kanjanavanit R, Sritara P, Krittayaphong R, Kunjara-Na-Ayudhya R, et al. Thai Registry in Acute Coronary Syndrome (TRACS) - An Extension of Thai Acute Coronary Syndrome Registry (TACS) Group: Lower In-Hospital but Still High Mortality at One-Year. J Med Assoc Thai. 2012;95:508.

2. ISIS-2 (Second International Study of Infarct Survival) Collaborative Group. Randomized trial of intravenous streptokinase, oral aspirin, both, or neither among 17,187 cases of suspected acute myocardial infarction: ISIS-2. Lancet. 1988;2(8607):349-60.

3. The GUSTO (Global Utilization of Streptokinase and Tissue Plasminogen Activator for Occluded Coronary Arteries) Investigators. An international randomized trial comparing four thrombolytic strategies for acute myocardial infarction. N Engl J Med. 1993;329(10): 673-82.

4. Van De Werf F, Cannon CP, Luyten A, Houbracken K, McCabe CH, Berioli S, et al. Safety assessment of single bolus administration of TNK-tPA in acute myocardial infarction: the ASSENT-I trial. Am Heart J. 1999;137(5):786-91

5. Van De Werf F, Adgey J, Ardissino D, Armstrong PW, Aylward P, Barbash G, et al. Single-bolus tenecteplase compared with frontloaded alteplase in acute myocardial infarction: the ASSENT-2 double-blind randomised trial. Lancet. 1999;354(9180):716-22.

6. The Assessment of the Safety and Efficacy of a New Thrombolytic Regimen (ASSENT)-3 Investigators. Efficacy and safety of tenecteplase in combination with enoxaparin, abciximab, or unfractionated heparin: the ASSENT- 3 randomised trial in acute myocardial infarction. Lancet. 2001;358(9282):605-13.
7. Hill MD, Barber PA, Takahashi J, Demchuk AM, Feasby TE, Buchan AM. Anaphylactoid reactions and angioedema during alteplase treatment of acute ischemic stroke. CMAJ. 2000;162(9):1281-4.

8. HPVC. Spontaneous reports of adverse drug reactions 2017. Bangkok: Graphic and Design; 2017.

9. Holloway K, Green T. Drug and therapeutics committees: A practical guide. Geneva, Switzerland: World Health Organization collaboration with Management Sciences for Health; 2013 [Available from: https://apps. who.int/medicinedocs/en/d/Js4882e/13.html]

10. Hughes D, Leethongdee $S$. Universal coverage in the land of smiles: lessons from Thailand's 30 Baht health reforms. Health Aff. 2007;26(4): 999-1008.

11. Betancourt BY, Marrero-Miragaya MA, Jimenez-Lopez G, Valenzuela-Silva C, Garcia-Iglesias E, Hernandez-Bernal F, et al. Pharmacovigilance program to monitor adverse reactions of recombinant streptokinase in acute myocardial infarction. BMC Clin Pharmacol. 2005;5:5.

12. The International Collaborative Study of Severe Anaphylaxis. Risk of anaphylaxis in a hospital population in relation to the use of various drugs: an international study. Pharmacoepidemiol Drug Saf. 2003;12(3):195-202.

13. The Gruppo Italiano per lo Studio della Streptochinasi nell'Infarto miocardico (GISSI). GISSI-2: a factorial randomised trial of alteplase versus streptokinase and heparin versus no heparin among 12,490 patients with acute myocardial infarction. Lancet. 1990;336(8707):65-71.

14. The ISIS-3 (Third International Study of Infarct Survival) Collaborative Group. ISIS-3: A randomised trial of streptokinase vs tissue plasminogen activator vs anistreplase and of aspirin plus heparin vs aspirin alone among 41,299 cases of suspected acute myocardial infarction. Lancet. 1992;339(8796):753-70.

15. The GUSTO Investigators. A comparison of reteplase with alteplase for acute myocardial infarction. N Engl J Med. 1997;337(16):1118-23.

16. Hill MD, Buchan AM. Thrombolysis for acute ischemic stroke: results of the Canadian Alteplase for Stroke Effectiveness Study. CMAJ 2005;172(10):1307-12.

17. Robles LA, Matilla AF. Brain Stem Ischemic Stroke Associated with Anaphylaxis. Cureus. 2018;10(3):e2289.

18. The GISSI. Effectiveness of intravenous thrombolytic treatment in acute myocardial infarction. Lancet. 1986;1(8478):397-402.

19. Buka RJ, Crossman RJ, Melchior CL, Huissoon AP, Hackett S, Dorrian S, et al. Anaphylaxis and ethnicity: higher incidence in British South Asians. Allergy. 2015;70(12):1580-7.

20. Zhao Y, Sun S, Li X, Ma X, Tang H, Sun L, et al. Drug-induced anaphylaxis in China: a 10 year retrospective analysis of the Beijing Pharmacovigilance Database. Int J Clin Pharm. 2018;40(5):1349-58.

21. Reber LL, Hernandez JD, Galli SJ. The pathophysiology of anaphylaxis. J Allergy Clin Immunol. 2017;140(2):335-48.

22. Tibbutt DA, Davies JA, Anderson JA, Fletcher EW, Hamill J, Holt JM, et al. Comparison by controlled clinical trial of streptokinase and heparin in treatment of life-threatening pulmonary embolism. Br Med J. 1974;1(5904):343-7.

23. A cooperative study. Urokinase-streptokinase embolism trial: Phase 2 results. JAMA. 1974;229(12):1606-13. 